\title{
Properties of the volume phase in the layerwise growth. Case of forming of new layer under effect of previous
}

\author{
R.Ye.Brodskii \\ State Scientific Institution "Institute for Single Crystals" of National \\ Academy of Sciences of Ukraine, Nauky av. 60, 61001 Kharkov, Ukraine
}

Received October 25, 2016

\begin{abstract}
It was studied in the work the properties of volume inclusions of transparent phase in an opaque sample, that grown layerwise. It was studied a case in which the regions of transparent phase of a new layer appear only above the regions of transparent phase of the previous layer. Numerical characteristics of this process - the average number $\langle n\rangle$ of "islands" of transparent phase in a new layer on one such "island" of previous layer. It was research the formation of regions of the transparent phase in separate layers and formation of through "windows of transparency" in the final sample. The law of changing of transparent phase area with the number of layer was received at different values of $\langle n\rangle$. Mechanisms, that determine the form of different parts of the law was pointed. For final sample the distribution density of "windows" on area was obtained, analytically for separate exactly solvable case and numerically in the general case. It is shown that the density has three different parts, the properties of these parts was studied at different $\langle n\rangle$. It was found the law of change of the total transparency of the sample with $\langle n\rangle$.

Keywords: Transparent phase, windows of transparency, total transparency of the
\end{abstract} sample.

В работе исследованы свойства объёмных включений прозрачной фазы в непрозрачном образце, выращенном послойно. Изучен случай, при котором области прозрачной фазы нового слоя зарождаются только над областьями прозрачной фазы предыдущего слоя. Числовая характеристика этого процесса - средниее число $\langle n\rangle$ "островков" прозрачной фазы в новом слое на один такой "островок" предыдущего слоя. Исследовано формирование областей прозрачной фазы в отдельных слоях и образование сквозных "окон прозрачности" в готовом образце. Получен закон изменения площади прозрачной фазы с номером слоя при различных значениях $\langle n\rangle$. Указаны механизмы, определяющие вид отдельных участков этого закона. Для готового образца получена плотность распределения "окон" по площади, аналитически в отдельном точно решаемом случае и численно в общем случае. Показано, что плотность имеет три различных участка, исследованы свойства этих участков при различных $\langle n\rangle$. Найден закон изменения суммарной прозрачности образца с $\langle n\rangle$.

Властивості об'ємної фази при пошаровому зростанні. Випадок формування нового шару під впливом попереднього. Р.Є.Бродський.

В роботі досліджені властивості об'ємних включень прозорої фази в непрозорому зразку, вирощеному пошарово. Вивчено випадок, при якому області прозорої фази нового шару зароджуються тільки над областю прозорою фази попереднього шару. Числова характеристика цього процесу - середнє число $\langle n\rangle$ "острівців" прозорої фази в новому шарі на один такий "острівець" попереднього шару. Досліджено формування областей прозорої фази в окремих шарах і утворення наскрізних "вікон прозорості" в 
готовому зразку. Отримано закон зміни площі прозорою фази з номером шару при різних значеннях $\langle n\rangle$. Вказані механізми, що визначають вид окремих ділянок цього закону. Для готового зразка отримана густина розподілу "вікон" по площі, аналітично в окремому точно вирішуваному випадку і чисельно в загальному випадку. Показано, що густина має три різних ділянки, досліджені властивості цих ділянок при різних $\langle n\rangle$. Знайдений закон зміни сумарної прозорості зразка з $\langle n\rangle$.

\section{Introduction.}

Let's consider a sample of the material grown in layers. Let each sample layer consists of a material of the main phase and, possibly, but not necessarily, the new phase regions. So a system with a complex phase distribution may be form ([1] - [4]). Herewith areas of a new phase in separate layers can be superimposed on each other and form a three-dimensional inclusions permeate the entire sample. The emergence of such an inclusion could qualitatively change the behavior of the sample. For example, if the main phase of the material - non-conductive and new phase conductive, the sample will conduct current. If the main phase is opaque, but a new phase - transparent, there will appear through "windows of transparency".

In this paper we investigate the formation of such "windows of transparency". Example of formation of "window" shown in Figure 1.

The position and the area of "windows of transparency" determine by the position and area of the region of transparent phase in separate layers. Processes of these areas forming can be divided into two classes: dependent on the phase distribution in previous layers and the independent. One of the cases of independent forming of the areas of transparency considered in [5]. In this paper we studied the formation of "windows of transparency" in the case where such a dependence presents. Namely, when the transparent areas in the new layer appear only above transparent areas in the previous layer.

\section{Statement of the Problem.}

Let the regions of transparency in the layer are originate at the beginning of the layer formation and grow evenly in all directions. We also assume that the time formation of all the layers are the same. Then all of these regions of transparency will be the circles of the same radius $r$. We call these elementary transparency regions "islands" of transparency, keeping the term "region of transparency" for connected associations such "islands". The layers of the sample will also be considered round (i.e. samples cylindrical) of radius $R$.

The origin of the new "islands" strictly above the regions of transparency of the previous layer means that above these areas the centers of the transparent circles appear. Except of this condition, no restrictions on the location of the points of origin of the "islands" apply. We assume that they are formed with equal probability at any point over the region of transparent phase of the previous layer.

The number of layers - $N$, layers are numbered from zero. This numbering is useful, because source layer is selected - "islands" of transparency in it are formed by a mechanism different from the mechanism of "islands" forming in the other layers. Because under it there is no previous layer "islands" should appear in it themselves. We assume that in the zero layer the specified number $k_{0}$ of "islands" originate at random points uniformly distributed in the layer.

The probability density $p$ of origin of new "island" above the given point of transparent region of previous layer $p=$ const. I.e. the probability of origin of "island" above the given element $\Delta S$ of transparent phase is equal to $p \Delta S$.

Note that because "islands" may overlap, the total area of the transparent phase in the layer can be less than the sum of the areas of individual "islands".

In the places of overlay of transparency regions of the all layers the "window of transparent" appear. We find the distribution of the "windows" on area. 


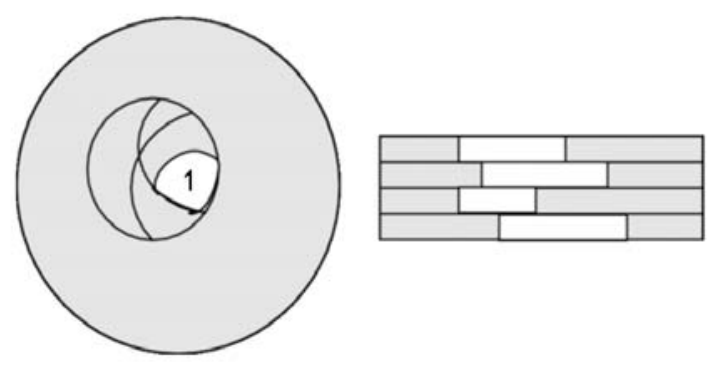

Fig. 1. An example of the formation of the "window of transparency" in place of superimposing of transparent areas in the individual layers. The case of four plates and one transparent area in each layer. Left top view, right side view. Digit 1 indicated "window of transparency".

Next consider the formation of regions of transparency in individual layers of the sample, and then the formation of "windows of transparency" in places of their intersection.

\section{Regions of transparency in the individual layers.}

Consider the problem of a change in sequential layers of the total area of the transparent phase.

Let the probability density of the formation of a new "island" of transparency above the transparent area of the previous layer is equal to $p$ and the area occupied by the transparent phase in the previous layer is equal to $S$. Let us find the probability $P_{k}$ of formation of given number $k$ of new "islands" of transparency in the layer and the average number $\langle k\rangle$ of new "islands".

Let's divide the area occupied by the transparent phase in the previous layer in the small elements $\Delta S$. In each element the "island" can be originated with probability $p \Delta S$ or don't originate with probability $1-p \Delta S$. Let $P_{k}$ - the probability of case, when with $\Delta S \rightarrow 0$ "islands" will originate at exactly $k$ elements, and nowhere else. All "islands" of transparency formed independently. The probability of the origin of one "island" of transparency anywhere over a transparent phase of previous layer is equal to $p S$. Probability $P_{k}$ is equal to, taking into account the permutations,

$$
P_{k}=\frac{(p S)^{k}}{k !} P_{0}
$$

where $P_{0}$ - the probability of any "island" will not originate.

$$
P_{0}=\lim _{\Delta S \rightarrow 0}(1-p \Delta S)^{\frac{S}{\Delta S}}=e^{-p S}
$$

and

$$
P_{k}=\frac{(p S)^{k}}{k !} e^{-p S}
$$

In addition it performs normalization condition $\sum_{k=0}^{\infty} P_{k}=1$. Average number $\langle k\rangle$ of new "islands" of transparency

$$
\langle k\rangle=\sum_{k=0}^{\infty} k \cdot \frac{(p S)^{k}}{k !} e^{-p S}=\sum_{k=1}^{\infty} \frac{(p S)^{k}}{(k-1) !} e^{-p S}=p S \cdot \sum_{k=0}^{\infty} \frac{(p S)^{k}}{k !} e^{-p S}=p S
$$

It is convenient to introduce the value of the average number of new "islands" on one "island" in the previous layer,

$$
\langle n\rangle=\frac{\langle k\rangle}{S / s_{0}}=p s_{0}
$$




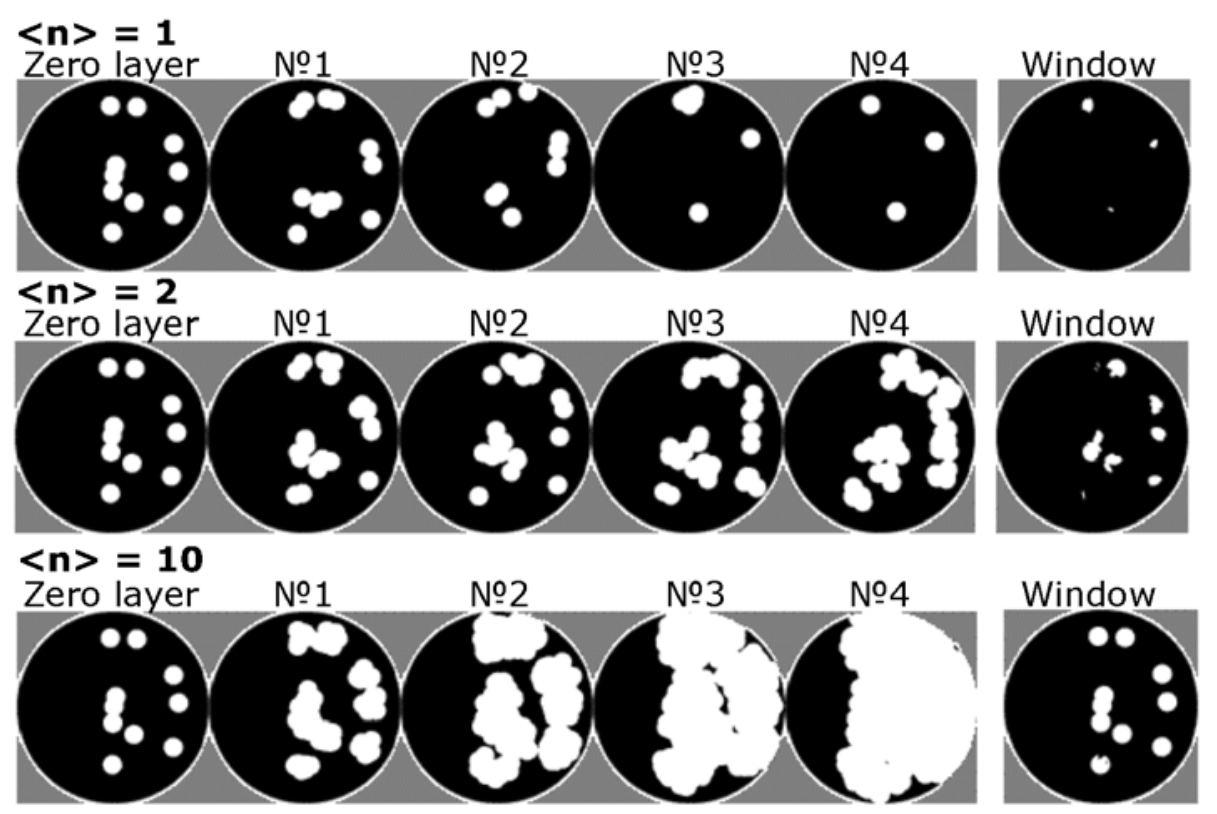

Fig. 2. Images of sample layers and the resulting "windows of transparency". The number of layers of $N=5$, the radii of the regions of transparency $r=0,1 R$. The average number of new "islands" on one "island" of the previous layer $\langle n\rangle=1,2,10$ (top to bottom).

where $s_{0}=\pi r^{2}=$ const - area of one "island". This value, as can be seen, is uniquely related to $p$ and in addition has a convenient obvious sense: each individual "island" of transparency in the previous layer will turn into a new layer to, in the average, $\langle n\rangle$ islands. In other words, if among new "islands" there is no the intersection, then the total area of the transparent phase in the new layer increases, in average, in comparison to the previous layer, exactly in $\langle n\rangle$ times. The value $\langle n\rangle$ is convenient to specify instead of the density $p$.

The type of changes of the area of transparent phase from layer to layer depends on $\langle n\rangle$. Namely, if the contribution of intersection of individual "islands" between themselves and with the boundaries of the layer is small, it should be expected that

1. At $\langle n\rangle<1$ transparent phase area in the layer will, in average, decrease with layer number, so in a some layer transparent phase will completely disappear. All next layers will be opaque and "windows of transparency" will not exist.

2. When $\langle n\rangle=1$ phase transparent area will not, in average, varied from layer to layer. Obvious assumptions about the shape and the area of "windows of transparency" in this case can not be done.

3. At $\langle n\rangle>1$ phase transparent area will, on average, grow with the number of layer, until it fills the entire layer. All next layers will be completely transparent (perhaps with slight fluctuations). If $\langle n\rangle$ is sufficiently large, then all the layers above the zero will be transparent in the places above the transparency regions of zero layer. In this case, "window of transparency" will be virtually the same as the transparency regions of zero layer.

Numerical simulation of the described process was performed. Figure 2 shows the image of $N=5$ sequential layers and formed by their covering "windows of transparency" for $\langle n\rangle=1,\langle n\rangle=2$ and $\langle n\rangle=10$. Initial number of "islands" $k_{0}=10$, their location in the zero layer was taken in all cases the same.

It can be seen that the simulation results confirm the assumptions made, but the "equilibrium" value of $\langle n\rangle_{e q}$, at which the total transparent phase area does not change from one layer to another, is not equal to one, and has the value $\langle n\rangle>1$. This is due to the presence of the intersection of individual "islands" with each other and with the layer boundaries. As a result of the intersection the total area of a transparent phase in layer is less than the sum of the areas of individual "islands". With chose parameters the equilibrium $\langle n\rangle_{e q}$, as seen in Figure 2, is between one (the total area decreases) and two (the total 

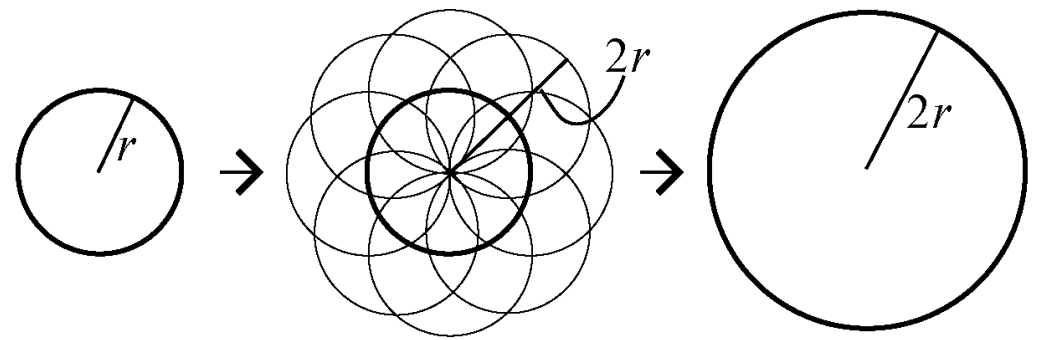

Fig.3. Forming of the region of transparency above the individual "island" in zero layer, $\langle n\rangle \rightarrow \infty$.

area increases). When $\langle n\rangle=10$ "windows of transparency" is really practically coincide with regions of transparency of zero layer.

The law of changing of the transparent phase area with a layer number

We assume that in the zero layer the transparent phase covers an area that small compared to the area of the layer. This is a typical case if we consider the opaque phase as the "basic" and transparent as the "extra". A small filling of zero layer by transparent phase means that the number $k_{0}$ of "islands" in the zero layer is small and the radius $r$ of individual "islands" is $r<<R$.

In this case, at $\langle n\rangle\left\langle\langle n\rangle_{e q}\right.$ the total area of the transparent phase, decreasing with the number of layer, rather quickly reaches zero. Because transparent phase consists of "islands", the law of variation of its area in this case will have the form of a small (no more than $k_{0}$ ) number of steps. When $\langle n\rangle=\langle n\rangle_{\text {eq }}$ law is trivial - the area does not change.

We next consider the case $\langle n\rangle\rangle\langle n\rangle_{e q}$. The total area of the transparent phase $S_{i}$ increases with the layer number $i$. Let's study the law of increase.

In the zero layer regions of transparency at low $k_{0}$ and $r$ are separated by a distance, on average, larger than the size of themselves. In this case, will be a three stage of changing of the area of $S_{i}$ from layer to layer:

1. Independent growth of the transparent phase around individual regions of transparency of zero layer.

2. Merge of the regions of transparency in clusters and intersection of these clusters with the layer boundary.

3. Go out to "sat uration" - full cover of layer by transparent phase and stop of further growth. Leaving only of small fluctuations in the area of the transparent phase directly "under" value $S_{i}=S_{\text {layer }}$.

Consider the first stage. Let "islands" of transparency in the zero layer lie far from each other and from the border. In the limit $\langle n\rangle \rightarrow \infty$ the regions of transparency in the next layer will be formed as shown in Figure 3

Thus, in the second layer transparent phase circle radius $2 r$ is formed, in the third layer - 3r etc. Transparent phase area in this case grows quadratically $S_{i}=k_{0} \cdot \pi((i+1) r)^{2}=k_{0} \cdot \pi r^{2}(i+1)^{2}, \quad i=0, \ldots$ - layer number.

At $\langle n\rangle \nrightarrow \infty$ ring with thickness $r$ around the initial round will not be fully occupied by the transparent phase. The region with rather complicated shape is formed, this case will be investigated further in the numerical simulation.

Consider the second stage. Suppose that in a certain layer transparent phase occupies a large area, much larger than that of a single "island" of transparency. This case takes place when many regions of transparency combine in one cluster. Let the perimeter of the cluster is equal to $l$. As above, take at first $\langle n\rangle \rightarrow \infty$. In this case, the transparent phase area increment in the next layer is approximately equal to $r \cdot l$. The perimeter changes on the value order of $\Delta l \sim r<<l$. Thus, in the following $\tilde{i}<<\frac{l}{r}$ layers transparent phase area growth will be close to the line.

Of course, a large cluster is formed finally and in the first stage of discussed above quadratic growth at high $i$. But in this case, "linear" growth will not mean anything more than a linear approximation of a quadratic law for large values of the argument. Linear law, which was discussed above, without "parabolic beginning", will be observed with fast, at two or three layers, the appearance of a new type of cluster. For 

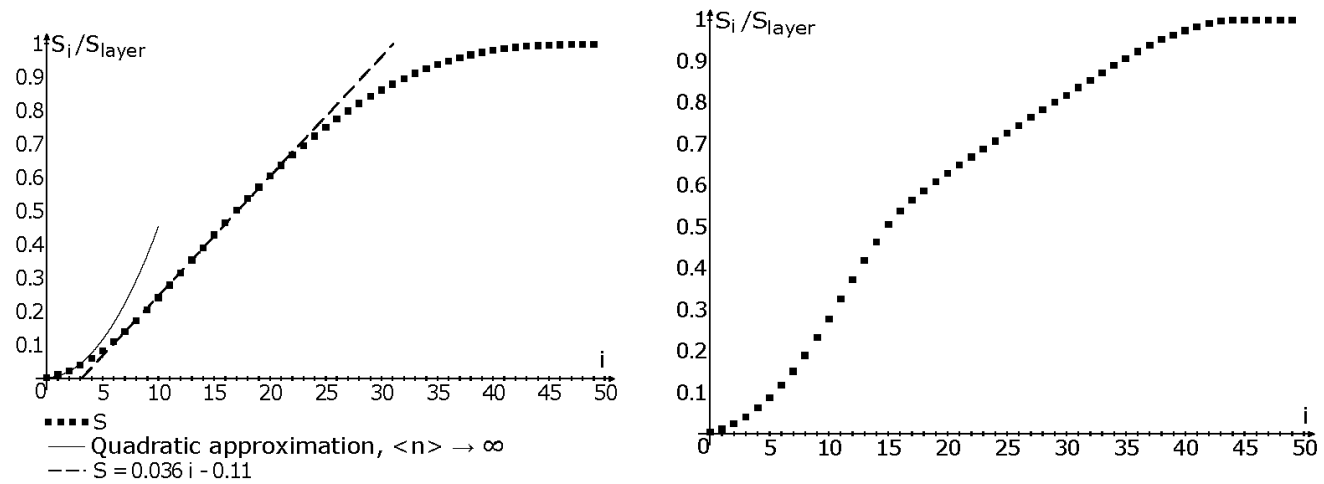

Fig. 4. The dependence of the transparent phase area from layer number, $r=0,03 R, k_{0}=5,\langle n\rangle=10$. Left - the average values for all performed experiments, to the right - a separate experiment.

example, when several individual transparent areas merging forming one cluster. Or when cluster grow up to the boundary of layer and thus significant portion of the perimeter of the cluster exclude of the cluster growth process.

In the third stage the layer will be almost completely covered by the transparent phase. The area of the transparent phase $S_{i}$ will have a value close to the area of the entire layer $S_{\text {layer }}$ and will only fluctuate due to the appearance at the finite $\langle n\rangle$ of small opaque areas in each new layer.

Let's move on to the numerical study of the law of change of transparent phase space with layer number at finite $\langle n\rangle$.

To study the all stages pointed above the numerical simulation was carried out at $r=0,03 R, k_{0}=5$. These values are small enough to make a noticeable all stages of the process. It was carried out 300 experiments, in experiments the location of regions of transparency in the zero layer was changed, the results were averaged. The sequential growth $N=50$ layers modelled. The value of $\langle n\rangle$ has been taken equal to $\langle n\rangle=10$. Figure 4 on the left shows the resulting plot of the depending of the total transparent phase area $S_{i}$ by layer number $i$. For convenience $S_{i}$ expressed as a part of the layer area.

It is seen that the results of experimental well express above three stages. In the first layers the transparent phase area varies according to the square law. In graph the portion of a parabola, given in the text, shown for the $\langle n\rangle \rightarrow \infty$, it is clear that the value $\langle n\rangle=10$ is enough to make for the first four layers the good coincidence of the law with the simulation results.

In the second step the transparent phase area varies according to the law, which is close to the line. The graph shows the straight line obtained as an approximation of the middle area. Slope of the line is 0.0357 . The dependence of this coefficient from $\langle n\rangle$ has also been carried out, the results are set out below.

It should be noted that at the different locations of the regions of transparency in the zero layer, the merging of individual regions in the total cluster begins with different numbers of layers. The same applies to the intersection of clusters with a layer boundaries. Thus, the moments of change of this regimes in the averaged results are not visible. To the right in Figure 4 the graph shows the results of a single experiment.

This graph clearly shows the points of stage change:

1. Transition of parabolic stage to linear stage of regions merge. This transition takes place approximately at the 11th layer. Image of 11th layer is shown in Figure 5 to the left. It is clearly seen the beginning of the merge process. (The graph have no a break there, a parabolic part is replaced by a straight-line smoothly.)

2. The transition from the cluster, which lies almost entirely within the layer, to the cluster, that went out on the layer border. In the graph it is the moment of change of the one linear part to the other, with the other slope. This transition takes place approximately at the 17th layer, picture of layer 17 is shown in Figure 5 in the middle.

3. Transition to the saturation regime, cessation of growth of area $S_{i}$ due the fact that the transparent 

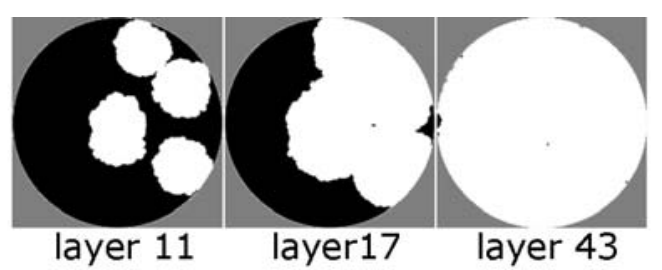

Fig. 5. Transient points in the law of variation of the transparent phase area with a number of layer. From left to right: the point of transition from quadratic regime of individual growth of regions to merging into clusters (layer 11), the point of transition to the cluster, intersects the boundary (layer 17), point of transition to saturation - full covering of layer by transparent phase (layer 43).
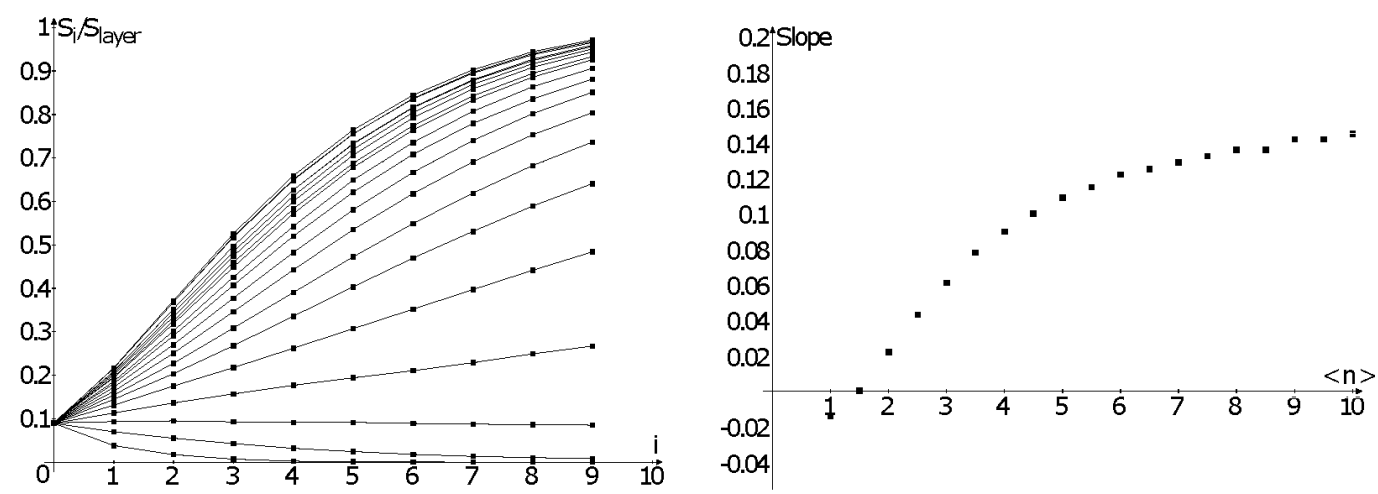

Fig. 6. Changing the transparent phase area with a layer number (left), $k_{0}=10, r=0,1 R, N=10$. $\langle n\rangle$ varies from 0.5 to 10 inclusively with step 0.5 . Graphs from the lowest to the highest correspond to change $\langle n\rangle$ from smallest to largest. The lines added for convenience. Right - a plot of the dependence of slope of the linear part on the $\langle n\rangle$.

phase already fills the entire layer. The transition occurs at approximately 43 layer. Image of 43 rd layer is shown in Figure 5 to the right. Small opaque areas visible on the left (growing of transparent phase, as can be seen from the previous illustration, was from right to left), and small opaque areas within the layer and near boundary, - the result of the above-mentioned fluctuations.

We now investigate the dependence of the slope of the linear part of the $\langle n\rangle$. It is expected that with increasing of $\langle n\rangle$ angle, i.e. the growth rate of $S_{i}$, will increase. There are no restrictions on the growth of the slope. Indeed, if the number of $k_{0}$ of "islands" in the zero layer and the radius $r$ of the individual regions are not small, then at the $\langle n\rangle \rightarrow \infty$ is possible that at the first step area will increase from an initial value to the area of the entire layer so that "linear" part will be substantially vertical.

It has been carried out the numerically simulation for 20 values of $\langle n\rangle$ - from 0.5 to 10 , inclusively, every 0.5. In the experiment it was taken values $k_{0}=10$ and $r=0,1 R$, that allowed us to obtain the necessary data with less than the above number of layers $N=10$.

Received dependencies $S_{i}$ on the layer number $i$ shown in Figure 6 on the left. For all $\langle n\rangle$ from one and above the linear region is clearly visible, for large $\langle n\rangle$ it can also see the beginning of the saturation stage.

Slopes of the linear part for different values of $\langle n\rangle$ are shown in Fig. 6 on the right. For $\langle n\rangle=1$ coefficient is less than zero, the area with the layer number decreases. When $\langle n\rangle=1.5$ area virtually unchanged. This means that the equilibrium value of $\langle n\rangle_{e q}$ close to 1.5. At larger $\langle n\rangle$ slope is greater than zero and increases with $\langle n\rangle$, as expected.

And a few more words about the general nature of the change (decreasing, conservation, increasing) of transparent phase area with layer number. Generally speaking, the equilibrium $\langle n\rangle_{e q}$ may be different for different layers. Simple limiting case: with the complete coating of layer by transparent phase, "equilibrium" is any value $\langle n\rangle$, when the transparent phase does not decrease. Thus, for a certain layer the equality $\langle n\rangle=\langle n\rangle_{e q}$ can be true, even if this equality does not true for the previous layers. Hence, 


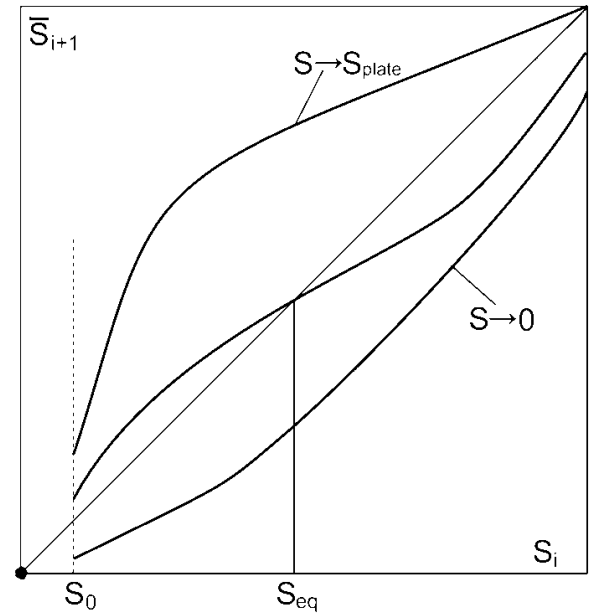

Fig. 7. The dependence of the average area of the transparent phase $\bar{S}_{i+1}$ in a new layer on its area $S_{i}$ in the previous layer. Both areas can have a value from zero to the entire area of the layer.

the dependence of the transparent phase area on the layer number can be not monotonous and more complicated.

Different cases of this dependence is convenient to study, considering the relation of average transparent phase area $\bar{S}_{i+1}$ in a new layer and its area $S_{i}$ (do not average, the exact value) in the previous layer. Some possible cases are shown in Figure 7 (the general course of the curves shown, not associated with a particular model).

Note that the curves have the boundary at the left, because $S_{i}$, unless it is zero, can not be less than $s_{0}=\pi r^{2}\left(\bar{S}_{i+1}\right.$ can be arbitrarily small). At that the point $(0,0)$ is always present, that is, between this isolated point and $S_{i}=s_{0}$ there is a gap outside the domain of definition.

The case $\langle n\rangle>\langle n\rangle_{e q}$ (for every $S_{i}$ ) corresponds to the curve that lies entirely above the diagonal, with the exception of the extreme right point of $S_{i}=S_{\text {layer }}$, where $\bar{S}_{i+1}=S_{i}$. Transparent phase area can not be larger than that of the layer. The case $\langle n\rangle\left\langle\langle n\rangle_{e q}\right.$ (for every $S_{i}$ ) corresponds to a curve under diagonal. The case $\langle n\rangle=\langle n\rangle_{e q}$ corresponds to the curve coincides with the diagonal.

In the general case, a graph of dependence of $\bar{S}_{i+1}$ from $S_{i}$ can cross the diagonal, does not coincide with it. These intersection points are points of equilibrium, that is, for such values of $S_{i}$ will be $\bar{S}_{i+1}=S_{i}$. If a curve crosses the diagonal from bottom to top, the point of intersection is a point of unstable equilibrium, if the curve crosses the diagonal from top to bottom (as in Figure 7), there will be a point of stable equilibrium. May be few intersection points, may also be intervals of coinciding of the curve with the diagonal.

\section{4. "Windows of transparency".}

Let's move on to the study of the process of "Windows of transparency" forming. "Windows" - the result of crossing of the transparent regions in all layers of the sample. We will seek the distribution density of the number of "windows" by the area and also total transparency, i.e. the part of the sample faces occupied by "windows".

First of all, consider the case solved exactly. In [5] it was shown that in the case of the independent formation of the regions of transparency in the different layers, it can get an accurate analytical solution for the case of two layers, in each of which one "island" of transparency is formed.

In the case studied in this paper, when new regions are emerging strongly above regions of the previous layer, this problem can be solved exactly too.

Let there is a sample consisting of two round layers with radius $R$. In first layer in a random location transparency circular region with radius $r$ is formed. We believe, as in [5], that the region is completely 


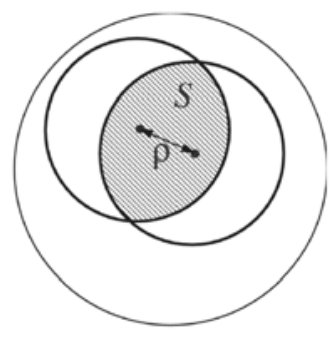

Fig. 8. Forming of the "window of transparency" for the $N=2$, one region of transparency in a layer.

within the layer, without crossing the boundary. Then, in the second layer at a random location above this region also one circular transparent region with radius $r$ emerging. In the place of their intersection "window of transparency" with area $S$ is formed (Figure 8).

Let's denote the distance between the centers of "islands" by $\rho$. Let the probability density of the origin of "island" in the second layer is equal to $p$. Note that the meaning of this density is different from what was discussed above. The higher the number of "islands" was arbitrary, so that $p \Delta S$ was the probability of origin of "island" of transparency over this element $\Delta S$ independently of the other elements, and $p$ may be set arbitrarily. Here we postulate the emergence of one and only one "island", so that $p \Delta S$ - the probability of that the "island" will arise over this, and only this element $\Delta S$. So that $p=\frac{1}{\pi r^{2}}$.

The probability density of the origin of "island" in the second layer at a distance of $\rho$ from the center of "island" in the first layer is equal to

$$
p_{\rho}(\rho)=\frac{2 \rho}{r^{2}} .
$$

Area $S$ forming at this case "window of transparency"

$$
S(\rho)=2 r^{2}\left(\arccos \frac{\rho}{2 r}-\frac{\rho}{2 r} \sqrt{1-\left(\frac{\rho}{2 r}\right)^{2}}\right), 0 \leqslant \rho \leqslant r
$$

The probability density of the formation of "window of transparency" with area $S$ is equal to $p_{S}=-p_{\rho}(\rho) \cdot \frac{d \rho}{d S}$ (the minus sign is associated with the fact that the area $S$ decreases with $\rho$ ).

$$
\frac{d \rho}{d S}=\frac{1}{\frac{d S}{d \rho}}=-\frac{1}{2 r \sqrt{1-\left(\frac{\rho}{2 r}\right)^{2}}},
$$

so that

$$
p_{S}(\rho)=\frac{2 \rho}{r^{2}} \cdot \frac{1}{2 r \sqrt{1-\left(\frac{\rho}{2 r}\right)^{2}}}=\frac{\rho}{r^{3} \sqrt{1-\left(\frac{\rho}{2 r}\right)^{2}}}
$$

Formulas (5) and (6) make up parametrically given expression for the density $p_{s}(S)$ of distribution of "windows" by area. In this case, in contrast to [5], we can not only get the parametric representation, but also write implicitly form. Express the $\rho$ from (6) and substituting in (5). We get

$$
S=2 r^{2}\left(\arccos \sqrt{\frac{\frac{p_{s} r^{2}}{2}}{\frac{p_{s} r^{2}}{2}+\frac{2}{p_{s} r^{2}}}}-\frac{1}{\left(\frac{p_{s} r^{2}}{2}+\frac{2}{p_{s} r^{2}}\right)}\right)
$$

The graph obtained density is shown in Figure 9. The minimum value of the area for which the density $p_{s}(S)$ determined, corresponds to the origin of new "island" on the border of the "island" in the first layer, $\rho=r$. The maximum value - to the origin in the center of the previous "island", $\rho=0, S=\pi r^{2}$.

In this case, the probability distribution density is the same as distribution density of the number of "windows", because the total number of "windows" every possible area equal to one, "window" by condition is one and only one. 


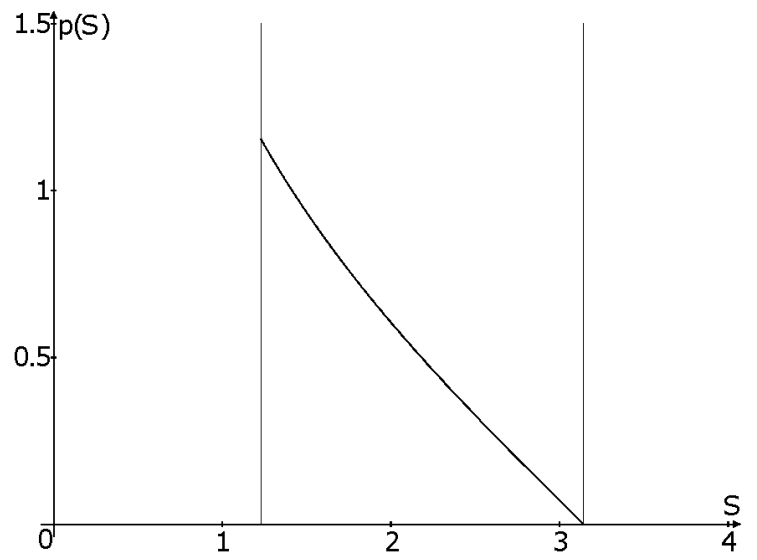

Fig. 9. The distribution density of the "windows of transparency" by area in the case of $N=2$, one transparency region in the layer, $r=1$.

Total transparency of the sample (total area occupied by the transparent phase) in this case, obviously, the same as a area of the single "window".

Assume now that "island" in the second layer may both arise and do not arise. Let $P_{1}$ - the probability that "island" will arise. In this case $p=P_{1} \cdot \frac{1}{\pi r^{2}}<\frac{1}{\pi r^{2}}$. As can be seen from the above calculations, changes in this case will consist in the fact that the $p_{s}$ receives multiplier $P_{1}$.

The general case. Let us return to the study of the formation of "windows of transparency" in general case. Above three cases of the process of forming the transparent phase in sequential layers was described. From the point of view of "windows of transparency" forming in these cases the following will happen.

1. At $\langle n\rangle\left\langle\langle n\rangle_{e q}\right.$ the filling of the layer by transparent phase decreases from layer to layer, and from a certain layer completely opaque layers are formed. In this case there will not the "windows of transparency". If the number of layers $N$ is not large enough for the complete disappearance of "windows", it should expect to appear only a small number of "windows" and with only small areas.

2. When $\langle n\rangle \approx\langle n\rangle_{e q}$ the area of transparent phase in the layers will be approximately conserved. In this case, it can expect the formation of "windows of transparency" with the very diverse areas and forms.

3. At $\langle n\rangle>\langle n\rangle_{e q}$ transparent phase area increases. If $\langle n\rangle$ is sufficiently large, the space over each region of transparency of the zero layer would be entirely filled with a transparent phase. As a result, "window of transparency" will coincide with the transparent regions of the zero layer. "Windows" will have an area equal or greater than $s_{0}=\pi r^{2}$ - area of separate "island" of transparency. If $\langle n\rangle$ is not large enough, the regions of transparency of the zero layer will partly overlap by opaque regions in the upper layers. In this case "windows" with an area a little less than $s_{0}$ will be formed, and possibly a some number of "windows" with small area $S<<s_{0}$.

We carried out numerical simulations of the process of "windows of transparency" forming by the mechanism presented above. We used the following parameters: the number of layers $N=5$, the radius of the "island" of transparency $r=0,1 R$, the number of "islands" in the zero layer $k_{0}=10$. Thus, the total area of the transparent phase in zero layer was equal to or less than (in the case of "islands" intersection) 0.1 from layer area. For obtaining of the distribution of "windows" by area it was carried out 300 experiments for each $\langle n\rangle$ in the range $\langle n\rangle=0,5 \ldots 10$ with step 0.5 . In this range of $\langle n\rangle$ with the chosen parameters all the cases listed above are implemented.

The simulation results are illustrated in Figure 10. It shows "windows of transparency" for $\langle n\rangle=$ $1,2, \ldots, 10$, and the same in all the experiments the original location "islands".

It can be seen that for small $\langle n\rangle$ transparency region of the zero layer significantly overlap by opaque areas in the following layers. At $\langle n\rangle=0,5$ in this experiment "windows of transparency" are not formed. With large $\langle n\rangle$ "windows" practically identical with transparent regions in the zero layer. 


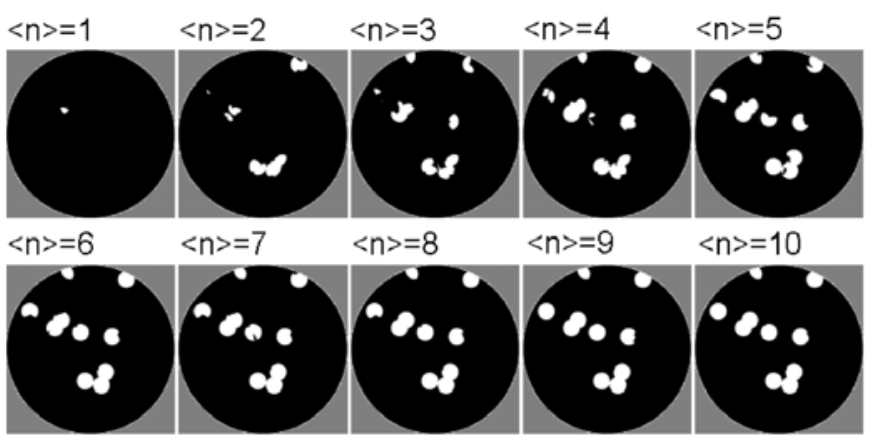

Fig. 10. "Windows of transparency" for $\langle n\rangle=1,2, \ldots, 10$ at $N=5, k_{0}=10, r=0,1 R$, for particular, one and the same in all the experiments, position of "islands" of transparency in the zero layer.
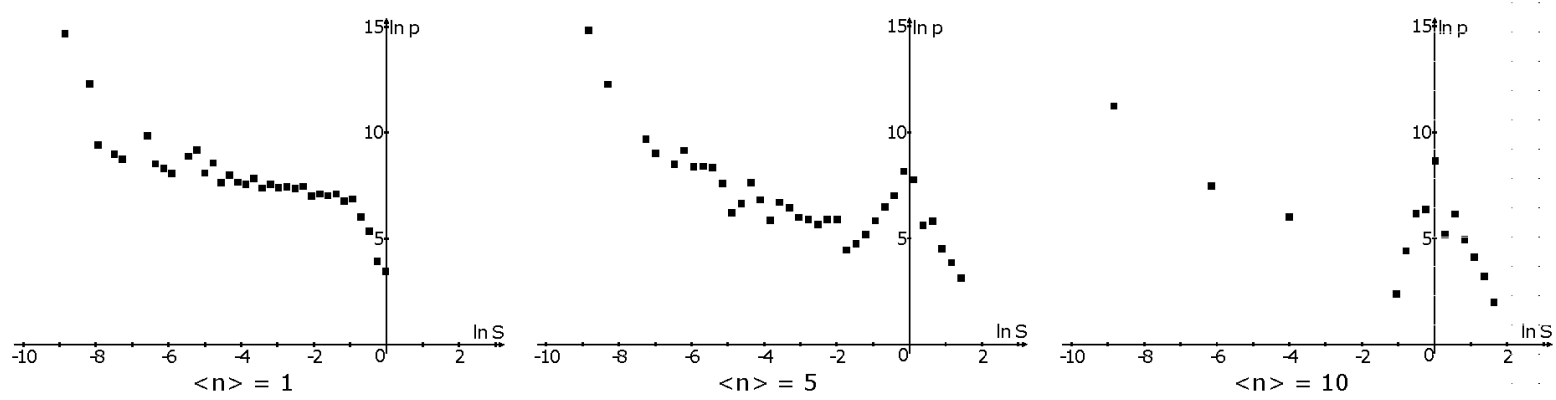

Fig. 11. The distribution density of the number of "windows of transparency" by area, three cases: small $\langle n\rangle=1$, the big $\langle n\rangle=10$, and the case of the general form $\langle n\rangle=5$. The areas specified as a fraction of $s_{0}$.

It should be noted that at a another location of "islands" in the zero layer noticeable coincidence of "windows" with regions of transparency in the zero layer could be achieved even at $\langle n\rangle \approx 5$.

According to the results of experiments graphs of the distribution density of the number of "windows" by area were constructed. Fig. 11 shows graphs for the case of small $\langle n\rangle=1$, the big $\langle n\rangle=10$ and a case of the general form $\langle n\rangle=5$. The graphs are given in double logarithmic scale. The areas on the horizontal axis, measured as a fraction of the $s_{0}$. Thus, the zero of horizontal (logarithmic) axis corresponds to the area of "window" equal to the area of the one "island".

It is seen that the distribution density have, in general, three sections. For $\langle n\rangle=5$ are clearly visible:

1. Descending part in large scales. The part is linear in two-log scale. This means that the distribution density in this area - power function. The part corresponds to "windows", the area of which more than $s_{0}$. Such "windows" may be formed only at the confluence of two or more "islands" of transparency in the zero layer

2. Increasing part in the middle scales, also linear in the double logarithmic scale. This part ends at the point $S=s_{0}$.

3. Descending part at small scales. Except for the "outliers"at a very small areas, also well approximated by a linear function in a double logarithmic scale.

From a comparison of graph for the case of $\langle n\rangle=5$ with graphs for small $\langle n\rangle=1$ and large $\langle n\rangle=10$ it's clear that

1. At small $\langle n\rangle$ part of medium scales disappears, and a large-scale part shifts to smaller $S$. When $\langle n\rangle=1$ points in region $S>s_{0}$ absent. This means that even consist of several "islands" transparent clusters in a zero layer overlap opaque areas in the upper layers so that the areas of the "windows" formed are less than one $s_{0}$.

2. At large $\langle n\rangle$ small-scale part contains a small number of points. However, it see clearly that these points lie on a straight line. 

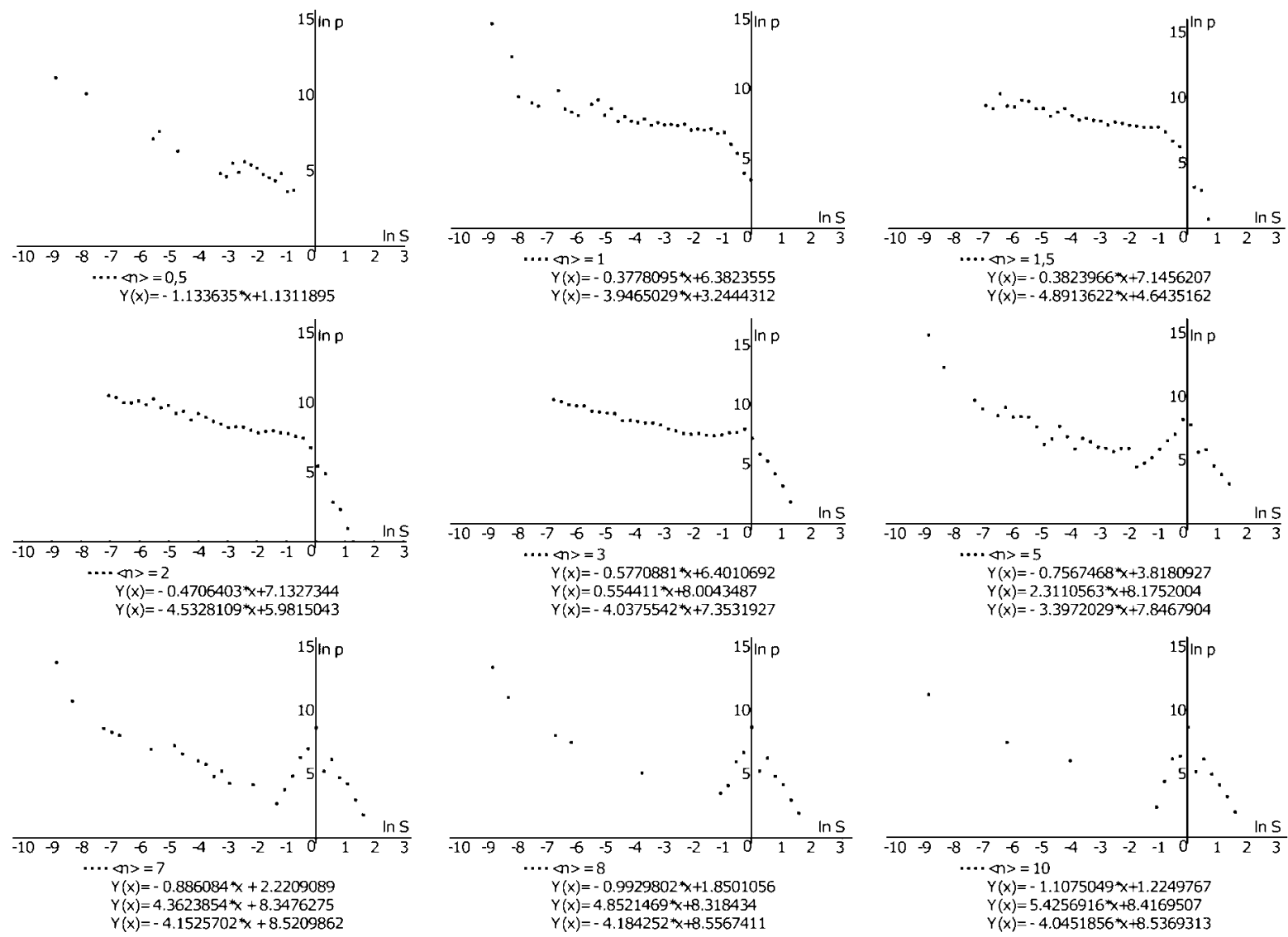

$Y(x)=-0.5770881 *+6.4010692$ $Y(x)=0.554411 * x+8.0043487$
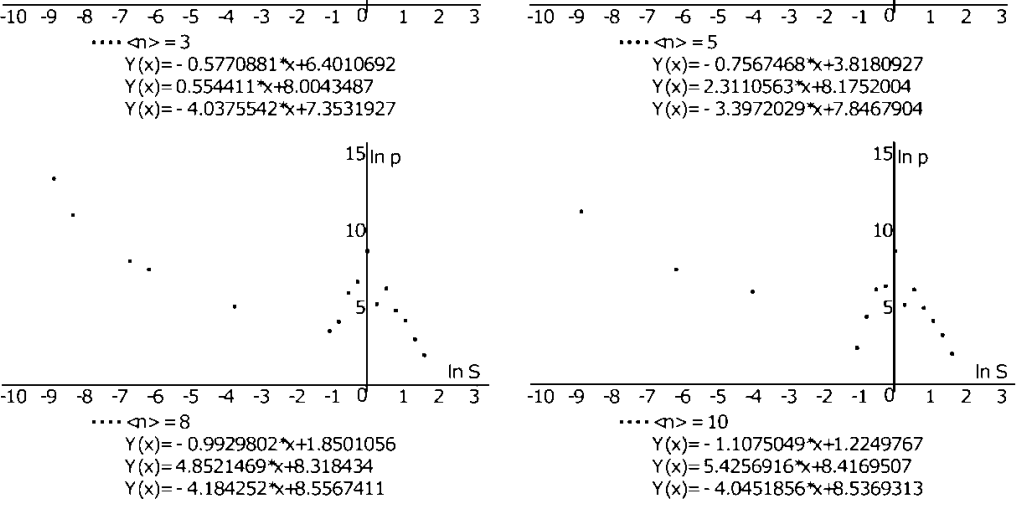

Fig. 12. Typical graphs of distribution density of "windows" by area for $\langle n\rangle$ from 0,5 to 10 .

Thus, distribution density $p(S)$ of "windows" by area has the form:

in small-scale region $p(S) \approx C_{1} S^{\alpha}, \alpha<0$,

in middle-scale region $p(S) \approx C_{2} S^{\beta}, \beta>0$,

in large-scale region $p(S) \approx C_{3} S^{\gamma}, \gamma<0$.

Let's investigate the dependence of the exponents $\alpha, \beta, \gamma$ from $\langle n\rangle$. Fig. 12 shows graphs of $p(S)$ for $\langle n\rangle$ in the range from 0.5 to 10 with linear approximations in different regions.

Values of exponents $\alpha, \beta, \gamma$ for all $\langle n\rangle$ from 0,5 to 10 with step 0,5 shown on the graph on figure 13 .

The graphs show that all three exponents behave differently when $\langle n\rangle$ smaller and larger then $\langle n\rangle_{0} \approx 5,5 \ldots 6$. Namely,

1. Exponent $\alpha$, except of outlier $\langle n\rangle=0,5$, has at $\langle n\rangle\left\langle\langle n\rangle_{0}\right.$ value near $-0,5$ and slow decreases. Follow at $\langle n\rangle \approx\langle n\rangle_{0}$ quickly decreases to -1 and follow at $\left.\langle n\rangle\right\rangle\langle n\rangle_{0}$ has value near -1 . Lines $y=-0,5$ and $y=-1$ for convenience, are shown on the graph.

2. Exponent $\beta$ in region $\langle n\rangle\left\langle\langle n\rangle_{0}\right.$ increases from values less than unity to about $4 \ldots . .5$. Then, when the $\langle n\rangle \approx\langle n\rangle_{0}$, this growth stops, and at the larger $\langle n\rangle$ exponent $\beta$ fluctuates around a constant (or slowly varying) value. The growth of $\beta$ on the left of the point $\langle n\rangle \approx 5,5 \ldots 6$ is close to the line. Straight line, found as an approximation to $\beta(\langle n\rangle)$ in this region has the form

$$
\beta(\langle n\rangle) \approx 1,264 \cdot\langle n\rangle-3,553 .
$$

3. Exponent $\gamma$ of large-scale region fluctuates near value $\gamma=-4$. At $\langle n\rangle\rangle\langle n\rangle_{0}$ this fluctuations are much smaller than at $\langle n\rangle\left\langle\langle n\rangle_{0}\right.$.

The reason for the different behaviour of exponents on the left and right from the point $\langle n\rangle=\langle n\rangle_{0} \approx$ $5,5 \ldots 6$ related to the fact that exactly at these values $\langle n\rangle$ "windows of transparency" start almost com- 


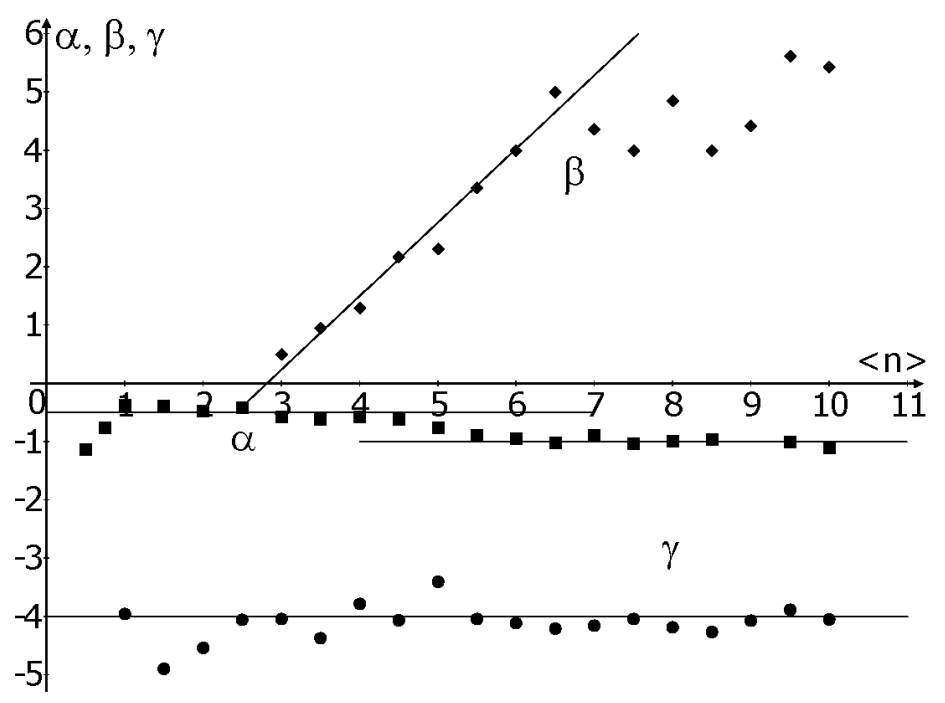

Fig. 13. Exponents $\alpha, \beta, \gamma$ for all $\langle n\rangle$ from 0.5 to 10 with step 0.5 . Increasing part of medium scales and, accordingly, the exponent $\beta$ exists for $\langle n\rangle \geqslant 3$. In the small-scale region $\langle n\rangle=0,75$ point is added. $\alpha$, exponent in the small-scale region, $-\beta$, exponent in the middle-scale region, $\bullet-\gamma$, exponent in the large-scale region.

pletely coincide with the transparent regions in the zero layer. Thus, when $\langle n\rangle$ smaller and larger then this point the distribution of "windows of transparency" by area is determined by different processes. To the left of this point - first of all, by the intersection of transparent regions in different layers, on the right - first of all, the union of transparent regions in the zero layer.

Note that the small-scale asymptotics obtained in [5] for the case of an independent formation of transparent phase in different layers, the same as obtained in the present work with $\langle n\rangle\left\langle\langle n\rangle_{0}\right.$. That is the condition for the appearance of new "islands" strictly above the transparent regions on the previous layer almost no effect on the small-scale asymptotic behaviour when $\langle n\rangle\left\langle\langle n\rangle_{0}\right.$.

Total transparency $S_{\text {sum }}$, i.e. the total area of "windows of transparency", as shown in Fig. 10, growing with $\langle n\rangle$, approaching the area of $S_{0}$ of transparent phase in zero layer. Fig. 14 shows a graph of total transparency, resulting in numerical simulation. For the convenience $S_{\text {sum }}$ is measured in fractions of the layer area. At selected for modelling $k_{0}=10$ and $s_{0}=0,01$ of layer area, the average area of the transparent phase in zero layer $\bar{S}_{0}$ has a value near 0.1 (slightly less due to the intersection of "islands").

\section{Conclusions.}

For the process of formation of transparent phase in the layers shown that there is an "equilibrium" value $\langle n\rangle=\langle n\rangle_{e q}$, when the transparent phase area $S_{i}$ does not change from layer to layer.

When $\langle n\rangle$ less than $\langle n\rangle_{e q}$, the area of the transparent phase decreases with layer number, "window of transparency", at a sufficiently large number of layers, are not formed. Numerical simulation of the process showed that $\langle n\rangle_{e q} \approx 1,5$.

At $\langle n\rangle$, larger than $\langle n\rangle_{e q}$, transparent phase area increases with the number of layer, the process pass at this the following steps:

a. Quadratic growth of $S_{i} \propto i^{2}, i$ - layer number. It corresponds to growth of the transparency regions around the individual "islands" of zero layer.

b. One or more linear parts. Each such part corresponds to the growth of clusters of its kind: the clusters formed from several merged regions of transparency; one common cluster without crossing a boundary of layer; cluster, part of which is "cut off" by the layer boundary. 


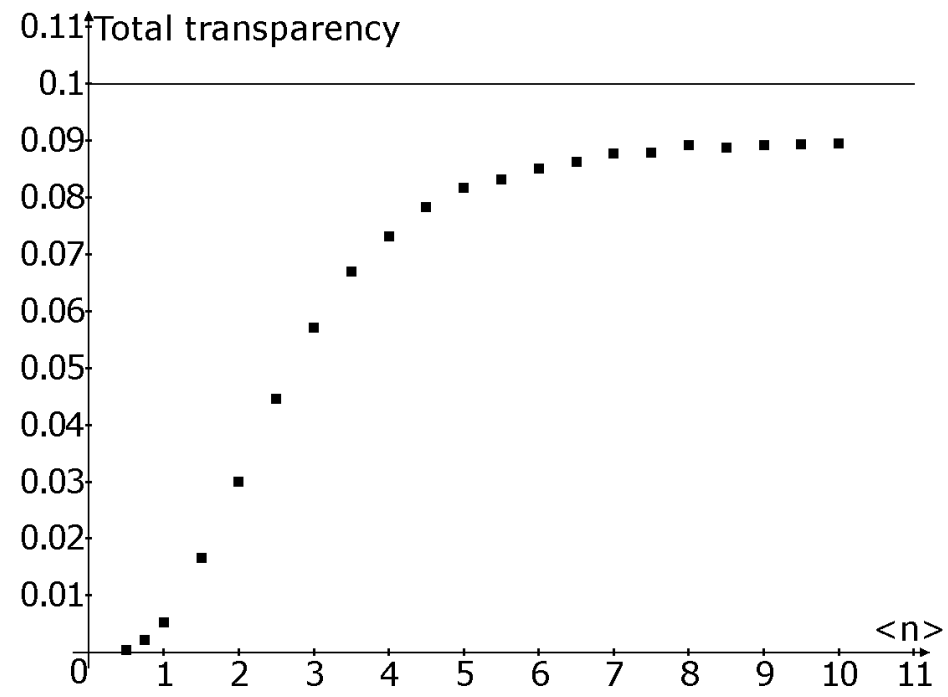

Fig. 14. The total transparency of the sample at $\langle n\rangle$ from 0,5 to 10 with step 0,5 (point 0,75 added).

c. Transition to saturation - approaching of the area of the transparent phase to the entire layer area. When fill the layer, the area ceases to grow, continue to fluctuate only.

Graphs of the transparent phase area $S_{i}$ are shown in Fig. 4 and 6. Figure 4 shows a detailed graph, it can see all stages of growth of the transparent phase. Fig. 6 left shows the set of graphs for $\langle n\rangle$ from 0.5 to 10 with step 0.5 , it can see changing of the slope of the linear part with $\langle n\rangle$. Fig. 6 on the right shows the graph of the slope by the $\langle n\rangle$.

2. The value of the equilibrium $\langle n\rangle_{e q}$ can, in general, depends on the filling of layer by transparent phase. Depending on the form of functions $\bar{S}_{i+1}\left(S_{i}\right)$ (Figure 7 ) a transparent phase area $S_{i}$ can change with the layer number $i$ is monotonously or have areas of decreasing, increasing and the points of equilibrium.

3. Distribution density $p_{s}(S)$ of "windows" by the area found analytically for a sample with two layers, each of which there is one and only one transparent region (Figure 8). In an implicit form:

$$
S=2 r^{2}\left(\arccos \sqrt{\frac{\frac{p_{s} r^{2}}{2}}{\frac{p_{s} r^{2}}{2}+\frac{2}{p_{s} r^{2}}}}-\frac{1}{\left(\frac{p_{s} r^{2}}{2}+\frac{2}{p_{s} r^{2}}\right)}\right),
$$

expression was also obtained in parametric form (formula (5), (6)). This dependence is shown in Figure 9 .

4. In the case of an arbitrary number of layers $N$ and an arbitrary number of "islands" in each layer distribution densities of "windows" by area were obtained by numerical simulation. Graphs of density at different $\langle n\rangle$ are shown in Fig. 12.

5. It is shown that the distribution density of the "windows" by area in general case has three parts - a decreasing small-scale part, medium-scale growing part and decreasing large-scale part. All three parts are well approximated by power functions (linear in log-log scale). The graphs of the exponents of approximation from $\langle n\rangle$ for all three parts are shown in Fig. 13.

6. All three part exhibit different behaviour at $\langle n\rangle$ smaller and larger than $\langle n\rangle \approx 5,5 \ldots 6$ :

a. Exponent of small-scale approximation at the left of this point has a value of approximately -0.5 , right - about -1 .

b. Exponent of approximation of medium-scales part at the left of this point increases linearly with a factor of approximately 1.264, right - fluctuates around a constant (or slowly varying) value.

c. Exponent of approximation of large-scale part and left and right has a value close to -4 , but to the left of this point it fluctuates considerably, while to the right little deviates from -4 . 
This behaviour relates to the fact that at the values of $\langle n\rangle>5,5 \ldots 6$, "windows of transparency" start to almost coincide with the transparent regions in the zero layer, while to the left from this point the intersection of transparent regions in different layers significantly affects to the formation of "window".

6. At $\langle n\rangle<5,5 \ldots 6$ approximation of the small-scale region coincides with the small-scale approximation of the case [5] the same and independent formation of transparent phase in different layers.

7. The end of medium-scale part and start of large-scale part is at the point $S=s_{0}$ at $\langle n\rangle>5$ and shifted to smaller $S$ at small $\langle n\rangle$. This means that a large-scale part corresponds to "windows" formed over the region of confluence of several "islands" of transparency in the zero layer.

8. The total sample transparency, i.e., the total area of "windows of transparency" increases with $\langle n\rangle$ and tends to the average area of the transparent phase in zero layer. The graph of total transparency is in Fig. 14 .

\section{References}

1. V.I.Trofimov, V.A.Osadchiy, Growth and morphology of thin films. Energoatomizdat, Moscow (1993) [in Russian].

2. B.Lewis, J.C.Anderson, Nucleation and Growth of Thin Films. Academic Press, New York (1978).

3. K.Oura, V.G.Lifshitz, A.A.Saranin, A.V.Zotov, M.Katayama, Introduction to surface physics. Nauka, Moscow (2006) [in Russian].

4. V.Barton, B.Kabrera, F.Frank, Elementary processes of crystal growth. IIL, Moscow (1959) [in Russian].

5. R.Brodskii, A.V.Tur, V.V.Yanovsky, Functional materials, 21(4), 448 (2014). 